\title{
miR-483-3p promotes the osteogenesis of human osteoblasts by targeting Dikkopf 2 (DKK2) and the Wnt signaling pathway
}

\author{
BIN ZHOU, KUN PENG, GUOQIANG WANG, WEIHUA CHEN, PING LIU, FEI CHEN and YIJUN KANG \\ Department of Spine Surgery, The Second Xiangya Hospital, Central University, Changsha, Hunan 410011, P.R. China
}

Received March 9, 2020; Accepted July 8, 2020

DOI: $10.3892 / \mathrm{ijmm} .2020 .4694$

\begin{abstract}
Osteoporosis is a systemic metabolic bone disease during which bone mass decreases and bone quality is reduced. Maintaining the bone formation capacity of osteoblasts is crucial for the treatment of osteoporosis. In the present study, bioinformatics analysis was performed on online microarray expression profiles to identify miRNA(s) related to osteoblast proliferation and bone marrow-derived mesenchymal stem cell (BMSC) osteogenic differentiation. The specific effects of candidate miRNAs on cell proliferation, osteogenic differentiation and Wnt signaling-related factors were examined. As regards the downstream mechanisms, online tools were employed to predict the downstream targets of candidate miRNAs and the predicted miRNA-mRNA binding was verified. Finally, the dynamic effects of miRNAs and mRNAs were examined. The results revealed that miR-483-3p expression was decreased in bone tissue samples from patients with osteoporosis. In miR-483-3p-overexpressing human osteoblasts, cell viability, DNA synthesis capacity and osteogenesis were promoted, and the protein levels of Wnt1, $\beta$-catenin and cyclin D1 were increased. However, the protein receptor activator of nuclear factor kappa-B ligand (RANKL)/osteoprotegerin (OPG) ratio and cell apoptotic rate were decreased. The Wnt signaling, antagonist Dikkopf 2 (DKK2), was targeted and negatively regulated by miR-483-3p. DKK2 knockdown exerted similar effects as miR-483-3p overexpression, while DKK2 overexpression inhibited cell viability, DNA synthesis capacity and osteogenesis. DKK 2 overexpression also decreased the Wnt $1, \beta$-catenin, and cyclin D1 protein levels, whereas it promoted the the RANKL/OPG ratio and the apoptosis of human osteoblasts. DKK2 overexpression reversed the functions of miR-483-3p overexpression. On the whole, the findings of the present study
\end{abstract}

Correspondence to: Dr Fei Chen or Dr Yijun Kang, Department of Spine Surgery, The Second Xiangya Hospital, Central University, 139 Renmin Road, Changsha, Hunan 410011, P.R. China

E-mail: chenfei1972@csu.edu.cn

E-mail: kangyijun@csu.edu.cn

Key words: osteoporosis, osteoblast, miR-483-3p, Dikkopf 2, Wnt signaling demonstrate that the miR-483-3p/DKK2 axis modulates the bone formation process by affecting osteoblast proliferation, pre-osteoblast differentiation into mature osteoblasts and new bone matrix formation.

\section{Introduction}

Osteoporosis, a systemic metabolic bone disease, which mainly occurs among elderly, thin, post-menopausal women (1-4), with the characteristic features of decreased bone mass, reduced bone quality and a deteriorated microarchitecture, which leads to an increased risk of bone fractures. The rate of bone resorption by osteoclasts overcomes the rate of bone formation by osteoblasts, which causes an imbalance in bone remodeling, thus leading to pathological bone disease (5). Therefore, effective strategies aimed at restoring bone formation capacity are required in order to develop effective treatments for osteoporosis (2).

For bone formation, osteoblast progenitors undergo proliferation and differentiation into mature osteoblasts. These mature osteoblasts subsequently lay down the bone matrix and mineralize it. Osteoblasts are produced by mesenchymal stem cell (MSC) precursors via several microenvironment signaling pathways (6-8), including the Wnt, transforming growth factor- $\beta$ (TGF- $\beta$ ) and bone morphogenetic protein (BMP) pathways, which are involved in the bone cell differentiation process and bone matrix synthesis. In canonical Wnt signaling, free (unbound) $\beta$-catenin translocates to the nucleus, where it binds to the T-cell specific transcription factor (TCF) and lymphoid enhancer-binding factor (LEF) to activate TGF- $\beta$ and BMP2 gene transcription. In addition, Wnt signaling enhances the transcription of osteoprotegerin (OPG) and decreases the level of receptor activator of nuclear factor kappa-B ligand (RANKL), thus determining a total decrease in the RANKL/OPG ratio, which suppresses the differentiation of osteoclasts and enhances overall bone formation (9). However, Wnt signaling is deregulated or even blocked by different molecules in osteoporosis. Dikkopf (DKK), Kremen and sclerostin factors inhibit LRP5/6 co-factor, whereas secreted frizzled related protein (sFRP) binds to Wnt factors. Therefore, the investigation of the inhibitory agents and mechanisms of Wnt signaling antagonists as well as the recovery of Wnt functions attenuate osteoporosis.

MicroRNAs (miRNAs or miRs) are a class of small, endogenous RNAs of 15-25 nucleotides (nts) in length. miRNAs 
modulate gene expression at the post-transcriptional level by targeting specific mRNAs for degradation or translational repression (10). With regard to osteoblasts and osteogenesis, miRNAs have been reported to target the mRNA of several regulators to elicit or hinder osteoblast differentiation, depending on the specific targets (11-13). Notably, several miRNAs affect osteogenesis by targeting Wnt signaling. For example, miR-29a binds to multiple negative regulatory factors of Wnt signaling, including Dkk1, Kremen2 and sFRP2, while its expression enhances bone formation (14). In addition, miR-218 is elicited through Wnt signaling and enhances bone formation, binding to multiple antagonists of Wnt signaling, including Dkk1, Sclerostin (SOST) and sFRP2 mRNA. The expression of these miRNAs forms a positive feedback loop that optimizes Wnt signaling to enhance osteoblast differentiation within various MSCs $(15,16)$. As a number of miRNAs have been found to be dysregulated in osteoporosis or during MSC osteogenic differentiation (17-19), it was hypothesized that there may be more miRNAs targeting Wnt signaling antagonists to promote osteoblast proliferation and osteogenesis.

Herein, multiple sets of online microarray expression profiles that report the differentially expressed miRNAs between normal bone and osteoporosis bone tissues, or between osteogenic differentiated bone marrow-derived MSCs (BMSCs) and non-differentiated BMSCs were downloaded and analyzed. The results of the analysis indicated that miR-483-3p may be associated with osteogenesis. Therefore, miR-483-3p was transfected into an immortalized human osteoblast cell line, hFOB1.19, to generate miR-483-3p-overexpressing cells in order to detect the specific functions of miR-483-3p overexpression on osteoblast proliferation and osteogenesis. Subsequently, online tools were used to search among Wnt signaling antagonists for the direct downstream targets of miR-483-3p, and DKK2 was predicted as the downstream target, and the potential binding of miR-483-3p to DKK2 was then validated. The specific role of DKK2 in osteoblast proliferation and osteogenesis was investigated. Finally, the dynamic effects of miR-483-3p and DKK2 were examined to investigate whether miR-483-3p exerts its effects by targeting DKK2. The present study thus demonstrates that the miR-483-3p/DKK2 axis modulates osteoblast proliferation and osteogenesis through $\mathrm{Wnt} / \beta$-catenin signaling. This axis may prove to be a potential target for the treatment of osteoporosis.

\section{Materials and methods}

Clinical sampling. A total of 6 normal bone tissues $(53.50 \pm 4.61$, female) were obtained from patients undergoing posterior lumbar interbody fusion (PLIF) at L4/5. In addition, 6 bone tissues were obtained from patients with osteoporosis [bone mineral density (BMD) T score $\leq-2.5,59.00 \pm 5.48$, female] undergoing PLIF at L4/5. During the process of the surgery, part of the spinous process from each patient was collected for tissue analysis. Osteoporosis was diagnosed by a BMD T score of $\leq-2.5$ at the lumbar or femoral neck by dual-energy X-ray absorptiometry (DXA) (20). No differences were observed in age, BMI, serum vitamin D status, serum calcium and parathyroid hormone (PTH) status between the controls and patients with osteoporosis. Written informed consents were obtained from all subjects. All clinical sampling was performed with the approval of the Institutional Review Board and Ethics Committee of the Second Xiangya Hospital [approval no. 2019(202)]. All experiments were conducted in accordance with the approved guidelines. Histopathological analysis was performed on bone tissue samples using hematoxylin \& eosin (H\&E) staining and Masson's staining following previously described methods (21).

$H \& E$ and Masson's staining of bone sections. All bone samples were decalcified using ethylenediamine tetraacetic acid (EDTA) for 3-5 days following 4\% formalin fixation and were then subjected to paraffin embedding, sectioning H\&E staining, and Masson's staining (Beyotime Institute of Biotechnology, Inc.) according to previously described methods (21). Three different fields of vision of each section were observed using an optical microscope (CKX53, Olympus Corporation) selected to calculate the mean length and thickness of the trabecula in each sample. During the decalcification process, the flexibility of all samples was evaluated daily and the hardness was assessed by needling until all samples were flexible and easily pierced by the needle (21).

Bioinformatics analysis and targeted gene prediction. GEO datasets (GSE74209, GSE115197 and GSE19232) were used for the analysis of miR-483-3p expression in osteogenesis-related samples. The lncTar (http://www.cuilab.cn/lnctar) online tool was used to predict the binding sites between miR-483-3p and 3'UTR of Wnt/ $\beta$-catenin signaling pathway-related genes.

Cell line and transfection. The human osteoblast 1.19 (hFOB1.19) (CRL-11372 ${ }^{\mathrm{TM}}$ ) immortalized human osteoblast cell line was obtained from ATCC and cultured in a 1:1 mixture of Ham's F12 Medium and Dulbecco's modified Eagle's medium supplemented with $2.5 \mathrm{mM}$ L-glutamine (without phenol red), $0.3 \mathrm{mg} / \mathrm{ml} \mathrm{G} 418$, and 10\% FBS (Invitrogen; Thermo Fisher Scientific, Inc.).

The modulation of miR-348-3p expression was achieved by transfection with $1 \mu \mathrm{g} / \mathrm{ml}$ miR-348-3p or anti-miR-348-3p vector (Shanghai GenePharma Co., Ltd.). miR-NC vector or anti-miR-NC vector was used as a control. DKK2 overexpression was achieved by transfection with a DKK2 overexpression vector (DKK2 OE; Shanghai GenePharma Co., Ltd.). The empty vector was used as a control. DDK2 knockdown was achieved by transfection with $20 \mathrm{nM}$ specific small interfering RNA (si-DKK2, sense, UAUCCUUUAUGUGUCAAACTT and antisense, GUUUGACACAUAAAGGAUATT, Shanghai GenePharma Co., Ltd.). si-NC was used as the negative control (sense, UUCUCCGAACGUGUCACGUTT and antisense, ACGUGACACGUUCGGAGAATT). All transfections were performed using Lipofectamine 3000 transfection agent (Invitrogen; Thermo Fisher Scientific, Inc.). After 48 h, the cells were harvested for use in further experiments.

Alizarin Red staining. hFOB1.19 cells were fixed in 4\% formaldehyde (Klinipath) for $10 \mathrm{~min}$ at room temperature, rinsed once with PBS, rinsed twice with deionized water, and stained 
with Alizarin Red solution (Sigma-Aldrich; Merck KGaA) for $40 \mathrm{~min}$ at $37^{\circ} \mathrm{C}$ to visualize calcium crystals in the matrix. Images were captured using a microscope (CKX53; Olympus Corporation) and analyzed using ImageJ software (NIH).

Reverse transcription quantitative-polymerase chain reaction $(R T-q P C R)$. Total RNA was extracted from target cells (transfected or untransfected) using TRIzol reagent (Invitrogen; Thermo Fisher Scientific, Inc.). DNA was eliminated by treating the extracted RNA sample with DNase I (RNase free; Invitrogen; Thermo Fisher Scientific, Inc.). First-strand cDNA was synthesized using oligo(dT)20 and Superscript II reverse transcriptase (Invitrogen; Thermo Fisher Scientific, Inc.). mRNA and miRNA expression levels were detected using SYBR-Green PCR Master Mix (Qiagen, Inc.). The qPCR thermocycling conditions were as follows: The initial denaturation was first performed at $95^{\circ} \mathrm{C}$ for 2 min followed by denaturation at $95^{\circ} \mathrm{C}$ for $15 \mathrm{sec}$ and annealing and extension at $60^{\circ} \mathrm{C}$ for $30 \mathrm{sec}$. The denaturation, annealing and extension were repeated for 40 circles. The expression of GAPDH (for mRNA expression) or RNU6B (for miRNA expression) was used as an endogenous control. The relative expression levels were calculated using the $2^{-\Delta \Delta \mathrm{Cq}}$ method (22). The primer sequences are listed in Table SI.

Cell counting kit-8 (CCK-8) assay for cell viability. The transfected or untransfected target cells were seeded in 96-well cell culture plates at a density of $1 \times 10^{4}$ cells $/ \mathrm{ml}$. Following $24 \mathrm{~h}$ of culture at $37^{\circ} \mathrm{C}, 10 \mu \mathrm{l}$ of CCK-8 agent (03285; Sigma-Aldrich; Merck KGaA) were added to each well to detect cell viability following a 2-h incubation at $37^{\circ} \mathrm{C}$. The optical density was determined at a wavelength of $450 \mathrm{~nm}$ using a microplate reader (VICTOR Nivo; PerkinElmer, Inc.).

5-Ethynyl-2'-deoxyuridine (EdU) assay of DNA synthesis capacity. At $24 \mathrm{~h}$ following transfection, a Click-iT ${ }^{\mathrm{TM}} \mathrm{EdU}$ Cell Proliferation kit (C10337; Thermo Fisher Scientific, Inc.) was used to stain transfected or untransfected cells according to the manufacturer's instructions. Cells were then imaged and counted under a microscope (CKX53; Japan Olympus Corporation).

Flow cytometric analysis of cell apoptosis. Cell apoptosis was analyzed by flow cytometry using an Annexin V-FITC/PI Apoptosis Detection kit (Beyotime Institute of Biotechnology, Inc.) according to previously described methods (23). Target cells (transfected or untransfected) in each group were cultured for $24 \mathrm{~h}$, digested, and then washed 3 times with PBS. Cells were then resuspended with Annexin V-FITC binding solution (195 $\mu \mathrm{l})$, and Annexin V-FITC (5 $\mu \mathrm{l})$ was then added. Propidium iodide staining solution $(10 \mu \mathrm{l})$ was then added, and the cells were incubated for an additional 10-20 min in the dark. Cell apoptosis was detected using CellQuest software (BD Biosciences, Inc.).

Western blot analysis of protein levels. Total proteins were extracted using RIPA lysis buffer (Beyotime Institute of Biotechnology, Inc.) from target cells (transfected or untransfected). The protein concentration was determined using a bicinchoninic acid protein assay kit (Beyotime Institute of
Biotechnology, Inc.) loaded (50 $\mu$ g per lane) on $10 \%$ sodium dodecyl sulfate (SDS)-polyacrylamide gels, and transferred onto PVDF membranes (Thermo Fisher Scientific, Inc.). The membranes were blocked for $2 \mathrm{~h}$ at $37^{\circ} \mathrm{C}$ with $5 \%$ non-fat milk in Tris-buffered saline with Tween-20 (TBST) and then incubated overnight at $4^{\circ} \mathrm{C}$ with the following primary antibodies: Ki-67 (dilution 1:1,000, 27309-1-AP; Wuhan Sanying Biotechnology), cleaved-caspase-3 (dilution 1:1,000, ab2302; Abcam), caspase-3 (dilution 1:1,000, 19677-1-AP; Wuhan Sanying Biotechnology), WNT1 (dilution 1:1,000, 27935-1-AP; Wuhan Sanying Biotechnology), $\beta$-catenin (dilution 1:1,000, 51067-2-AP; Wuhan Sanying Biotechnology), cyclin D1 (dilution 1:1,000, 60186-1-Ig; Wuhan Sanying Biotechnology), DKK2 (dilution 1:1,000, CSB-PA08279A0Rb; CUSABIO), RANKL (dilution 1:1,000, sc-9073, Santa Cruz Biotechnology, Inc.), OPG (dilution 1:1,000, sc-11383, Santa Cruz Biotechnology, Inc.) and GAPDH (dilution 1:3,000, T0004; Affinity Biosciences). The membranes were then incubated with an HRP-conjugated secondary antibody (dilution 1:5,000, SA00001-1 or SA00001-2, Wuhan Sanying Biotechnology) for $1 \mathrm{~h}$ at $37^{\circ} \mathrm{C}$ and then coated with ECL luminescence reagent (Perkin-Elmer Inc.). GAPDH was used as an internal normalization control.

Luciferase reporter assay for miR-483-3p binding to DKK2 3'-UTR. The DKK2 3'-UTR was amplified by PCR and cloned into downstream of the Renilla psiCHECK2 vector (Promega Corporation), and named wt-DKK2 3'-UTR. The seed region of the DKK2 3'-UTR containing the predicted miR-483-3p binding site was mutated to construct the mutant vector (mut-DKK2 3'-UTR). These reporter vectors were then co-transfected into $293 \mathrm{~T}$ cells (ATCC) with miR-483-3p or anti-miR-483-3p and the luciferase activity was determined at $48 \mathrm{~h}$ following transfection using the Dual Luciferase Reporter assay system (Promega Corporation). Renilla luciferase activity was normalized to Firefly luciferase activity for each transfected well.

Data processing and statistical analysis. Data processing and statistical analysis were conducted using GraphPad software. Data are presented as the means $\pm \mathrm{SD}$, representing the results from at least 3 independent experiments. Differences between 2 groups were analyzed for statistical comparison using a Student's t-test. Differences between $>2$ groups were estimated for statistical comparisons using one-way ANOVA followed by Tukey's multiple comparisons post hoc test. Pearson's correlation coefficient analysis were used for correlation analysis. $\mathrm{P}<0.05$ was considered to indicate a statistically significant difference.

\section{Results}

Selection and verification of $m i R-483-3 p$. By cross-checking the data from several sets of online microarray expression profiles, it was found that miR-483-3p may be closely related to the osteoblast proliferation and osteoblast differentiation of BMSCs. According to GSE74209, the expression of miR-483-3p was significantly decreased in the femur tissue samples of patients with osteoporosis compared to those in normal bone tissue samples (Fig. 1A). According to GSE115197, the 
A

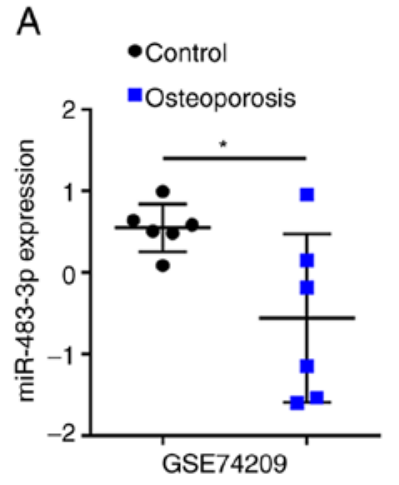

B

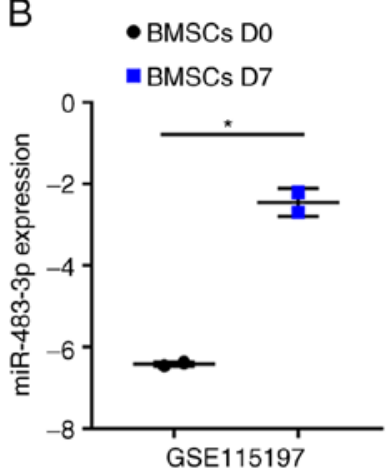

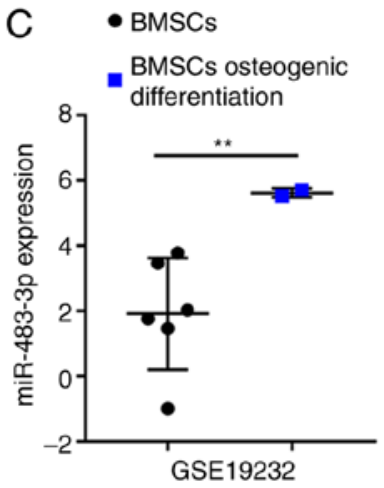

D H\&E
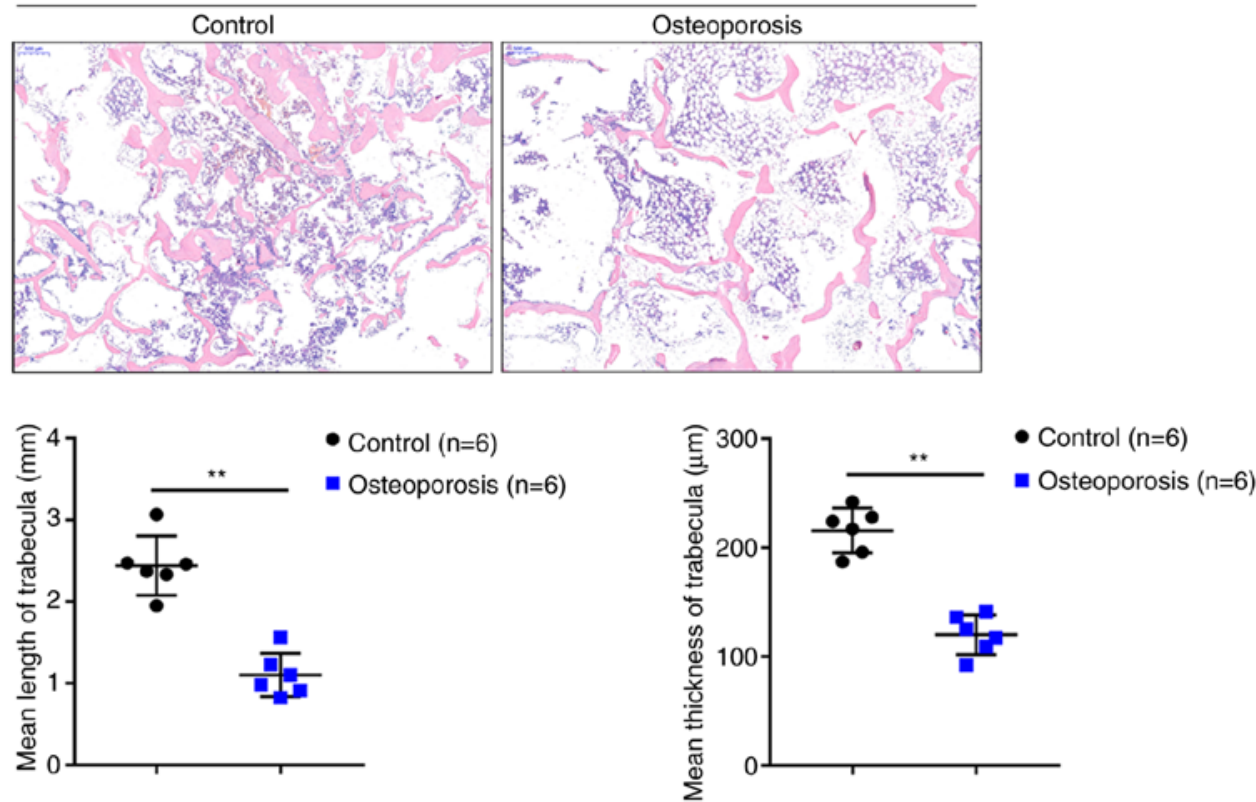

E

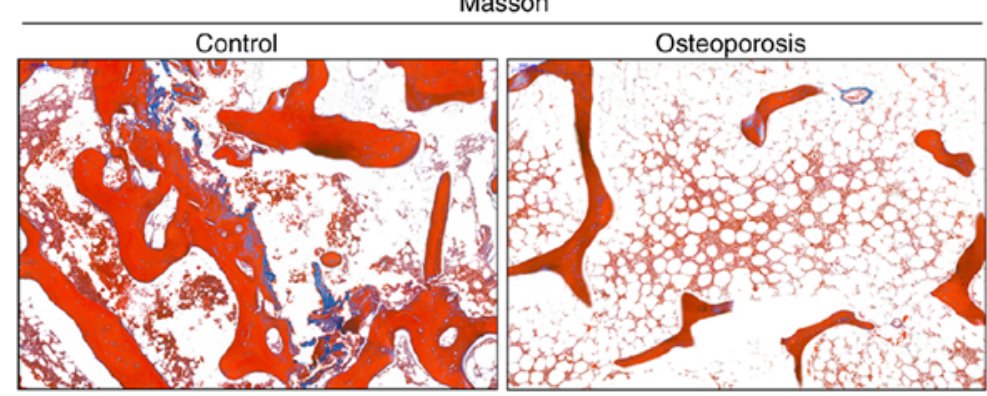

$\mathrm{F}$

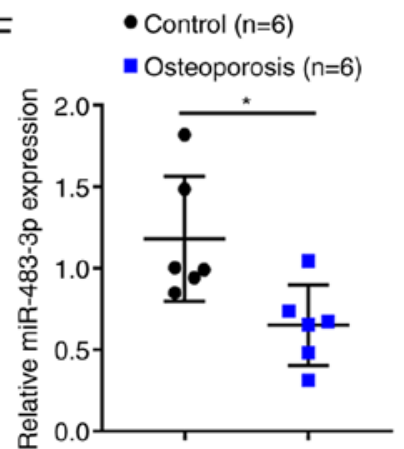

Figure 1. Selection and verification of miR-483-3p. (A) Expression of miR-483-3p in femur tissues of patients with osteoporosis and normal bone tissues according to GSE74209. (B) expression of miR-483-3p in the osteoblast precursors, bone marrow-derived mesenchymal stem cells (BMSCs), on days 0 and 7 of osteogenic differentiation induction according to GSE115197. (C) Expression of miR-483-3p in osteogenically differentiated or undifferentiated BMSCs according to GSE19232. (D and E) Representative images of H\&E staining and Masson's staining of normal bone tissues and osteoporotic bone tissues. (F) Expression of miR-483-3p in 6 normal bone tissues and 6 osteoporotic bone tissues determined by RT-qPCR. ${ }^{*} \mathrm{P}<0.05,{ }^{* *} \mathrm{P}<0.01$

expression of miR-483-3p was significantly increased in the BMSCs following 7 days of osteoblast differentiation induction compared to day 0 (Fig. 1B). According to GSE19232, the expression of miR-483-3p was significantly increased in osteogenically differentiated BMSCs compared to undifferentiated BMSCs (Fig. 1C). In summary, the expression of miR-483-3p was found to be downregulated in osteoporosis, in which osteogenesis was impaired; however, miR-483-3p expression was upregulated in osteogenically differentiated BMSCs.

To further examine miR-483-3p expression in osteoporosis, osteoporosis and normal lumbar bone tissue samples were obtained. Representative images of H\&E and Masson's staining of the osteoporosis and normal lumbar bone tissues are shown in Fig. 1D and E. H\&E staining revealed thinner and decreased trabecular bone in the osteoporotic bone tissues compared to 
the normal bone tissues (control group) (Fig. 1D). Moreover, the collagen in osteoporosis bone tissues was lightly colored and was sparsely arranged compared to normal bone tissues (control group) (Fig. 1E). Consistent with the above-mentioned online data, $\mathrm{miR}-483-3 \mathrm{p}$ expression was found to be decreased in 6 osteoporotic bone tissues compared to 6 normal bone tissues (Fig. 1F). Taken together, these findings suggest that miR-483-3p may contribute to the pathogenesis of osteoporosis in a osteogenic differentiation-related manner.

miR-483-3p plays a role in $h F O B 1.19$ cell proliferation and osteogenic differentiation. To examine the specific effects of miR-483-3p on osteoblasts, miR-483-3p was transfected into osteoblasts to generate miR-483-3p-overexpressing hFOB1.19 cells and RT-qPCR was performed to verify the transfection efficiency (Fig. 2A) and the related indexes. As regards osteoblast proliferation, miR-483-3p overexpression significantly promoted cell viability (Fig. 2B) and DNA synthesis capacity (Fig. 2C); however, it inhibited cell apoptosis (Fig. 2D). In addition, the protein level of the proliferation marker, $\mathrm{Ki}-67$, was increased, while that of the apoptosis-related cleaved caspase-3/caspase-3 ratio was decreased following the overexpression of miR-483-3p (Fig. 2E). As regards osteoblast osteogenesis, miR-483-3p overexpression significantly decreased the bone resorption-related factor RANKL/OPG ratio (Fig. 2E); however, it upregulated osteogenesis marker-related mRNA expression, including alkaline phosphatase (ALP), runt-related transcription factor 2 (RUNX2), and osteocalcin (OCN) (Fig. 2F). In addition, miR-483-3p overexpression significantly increased the Wnt1, $\beta$-catenin and cyclin D1 protein levels (Fig. 2E). Alizarin Red staining revealed that DKK2 knockdown increased hFOB1.19 cell mineralization (Fig. 2G) suggesting that miR-483-3p may enhance osteoblast proliferation and osteogenesis via $\mathrm{Wnt} / \beta$-catenin signaling.

miR-483-3p directly binds to DKK2. Considering that miR-483-3p regulates Wnt1, $\beta$-catenin and cyclin D1 protein levels, the lncTar online tool was employed to predict the downstream targets of miR-483-3p in the Wnt/ $\beta$-catenin signaling pathway. Following a cross-check, DKK2 was predicted by all the online tools as a direct downstream target of miR-483-3p (Fig. 3A). Before investigating the specific effects of DKK2 on osteoblast proliferation and osteogenesis, DKK2 expression was first examined in 6 normal bone tissues and 6 osteoporotic bone tissues. In contrast to miR-483-3p expression, DKK2 expression was significantly increased in the osteoporotic bone tissue samples (Fig. 3B). In the tissue samples, Pearson's correlation analysis revealed a negative correlation between miR-483-3p and DKK2 expression (Fig. 3C). To confirm the regulation of DKK2 by miR-483-3p, miR-483-3p/anti-miR-483-3p we transfected into the cells to generate cells with miR-483-3p overexpression or inhibition and RT-qPCR was then performed to verify the transfection efficiency (Fig. 3D). Consistent with their negative correlation, miR-483-3p overexpression decreased DDK2 protein expression, while miR-483-3p inhibition increased DKK2 protein expression (Fig. 3E).

The putative binding of miR-483-3p to DKK2 was then verified using a luciferase reporter assay. Two different types of DKK2 3'-UTR reporter vectors were constructed, wild-type (wt-DKK2 3'-UTR) and mutant (mut-DKK2 3'-UTR) (Fig. 3F). These vectors were co-transfected into 293T cells with miR-483-3p/anti-miR-483-3p, and examined for luciferase activity. miR-483-3p overexpression decreased wt-DKK2 3'-UTR luciferase activity, whereas miR-483-3p inhibition increased wt-DKK2 3'-UTR luciferase activity. In the mut-DKK2 3'-UTR group, the mutation of the predicted miR-483-3p-binding site eliminated the miR-483-3p-induced alterations in luciferase activity (Fig. 3F). In summary, these findings demonstrate that miR-483-3p targets DKK2 to decrease the DKK2 protein level.

Effects of DKK2 on hFOB1.19 cell proliferation and osteogenic differentiation. As miR-483-3p targets to inhibit DKK2, it was hypothesized that DKK2 knockdown may play a similar role to that of miR-483-3p overexpression in osteoblast proliferation and osteogenesis. Therefore, si-DKK2 was transfected into the hFOB1.19 cells to generate cells in which DKK2 was knocked down and RT-qPCR was then performed to verify the transfection efficiency (Fig. 4A). As was expected, DKK2 knockdown significantly enhanced cell viability and DNA synthesis capacity, while it inhibited cell apoptosis (Fig. 4B-D). Consistently, the expression of the Ki-67 proliferation marker was increased, whereas the cleaved-caspace3/caspase-3 apoptotic ratio was decreased by DKK2 knockdown (Fig. 4E). As regards osteogenesis, DKK2 knockdown significantly decreased the bone resorption-related factor, RANKL/OPG ratio (Fig. 4E), whereas it increased osteogenesis marker mRNA expression, including ALP, RUNX2 and OCN (Fig. 4F). Since DKK2 functions as a Wnt/ $\beta$-catenin signaling antagonist, DKK 2 knockdown significantly increased the Wnt1, $\beta$-catenin and cyclin D1 protein levels (Fig. 4E). Alizarin Red staining revealed that DKK2 knockdown increased hFOB1.19 cell mineralization (Fig. 4G), suggesting that DKK2 knockdown also promotes osteoblast proliferation and osteogenesis through Wnt $/ \beta$-catenin signaling.

Dynamic effects of miR-483-3p and DKK2 on hFOB1.19 cell proliferation and osteogenic differentiation. To investigate whether miR-483-3p exerts its functions by targeting DKK2, the dynamic effects of miR-483-3p and DKK2 on osteoblast proliferation and osteogenesis were examined. hFOB1.19 cells were co-transfected with miR-483-3p and DKK2 OE, and the related indexes were determined. The effects of miR-483-3p overexpression were similar to the above-mentioned observations (Fig. 5A-E). DKK2 overexpression significantly inhibited both cell viability and DNA synthesis, but promoted cell apoptosis (Fig. 5A-C). Regard to osteogenesis, DKK2 overexpression increased the RANKL/OPG ratio (Fig. 5D) but decreased the mRNA levels of ALP, RUNX2, and OCN (Fig. 5E). Consistent with these cellular functions, DKK2 overexpression decreased the Ki-67, Wnt1, $\beta$-catenin and cyclin D1 protein levels, whereas it increased the cleaved-caspase-3/caspase-3 ratio and DKK2 protein levels (Fig. 5D). Alizarin Red staining revealed that miR-483-3p overexpression increased hFOB1.19 cell mineralization, which was decreased by DDK2 overexpression (Fig. 5F). Taken together, these data demonstrate that DKK2 overexpression attenuates the functions of miR-483-3p overexpression, suggesting that miR-483-3p targets DKK2 to promote osteoblast proliferation and osteogenesis. 

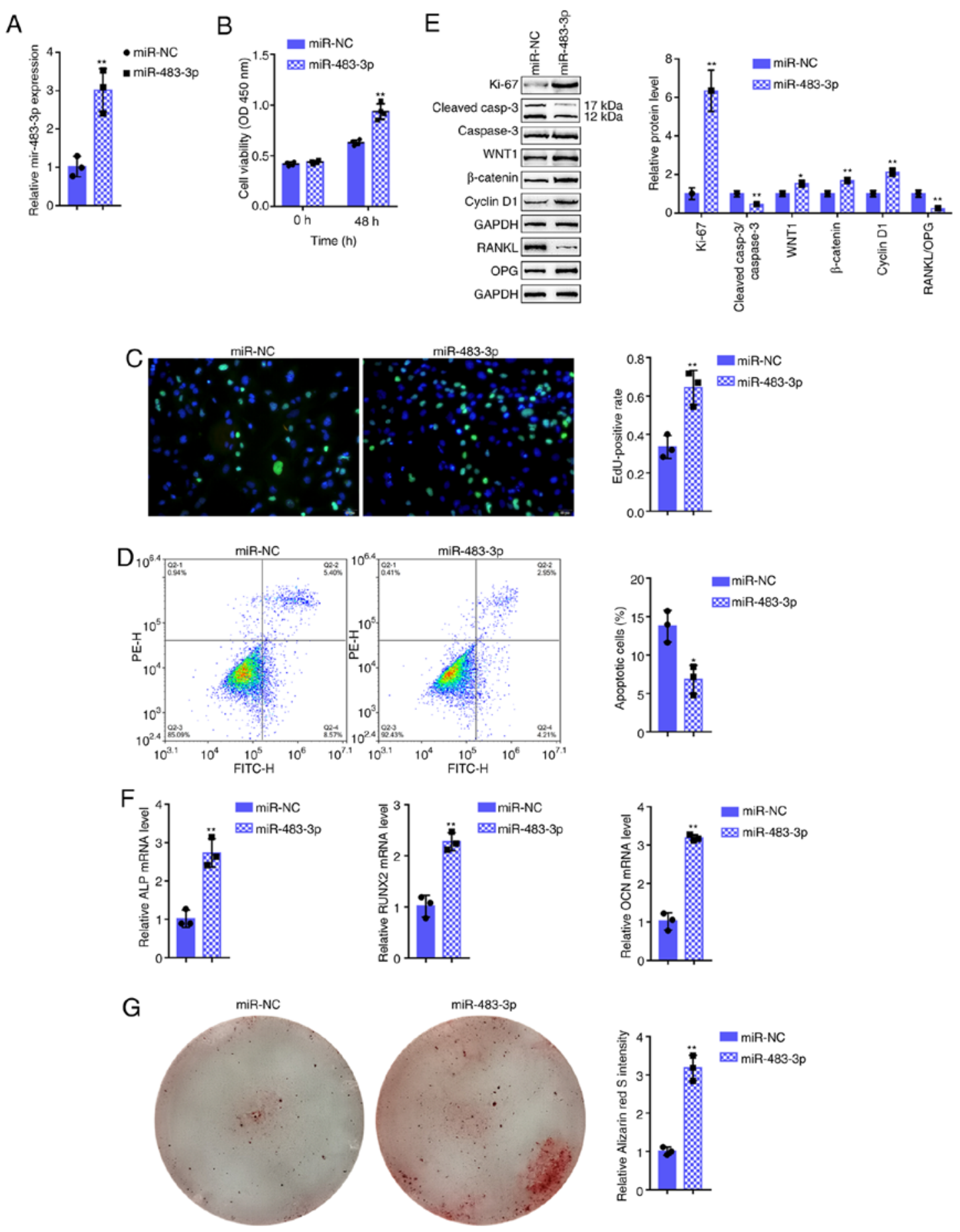

Figure 2. Effects of miR-483-3p on hFOB1.19 cell proliferation and osteogenic differentiation. (A) miR-483-3p overexpression was generated in hFOB1.19 cells by transfection with miR-483-3p, as confirmed by RT-qPCR. hFOB1.19 cells were transfected with miR-483-3p and examined for (B) cell viability by CCK-8 assay; (C) DNA synthesis capacity by EdU assay, and (D) cell apoptosis by flow cytometric assay. (E) The protein levels of Ki-67, cleaved-caspase-3, caspase-3, WNT1, $\beta$-catenin, cyclin D1, RANKL and OPG were examined by western blot analysis, and (F) the mRNA expression of osteogenesis markers, including ALP, RUNX2, OCN and OPN was determined by RT-qPCR. (G) The mineralization of hFOB1.19 cells was determined by Alizarin Red staining. ${ }^{*} \mathrm{P}<0.05,{ }^{* *} \mathrm{P}<0.01$.

\section{Discussion}

The present study demonstrated that miR-483-3p expression was considerably decreased in osteoporotic bone tissue samples. In miR-483-3p-overexpressing human osteoblasts, cell viability, DNA synthesis capacity and osteogenesis were promoted. Moreover, the Wnt1, $\beta$-catenin and cyclin D1 protein levels were increased, whereas cell apoptosis was inhibited in miR-483-3p-overexpressing human osteoblasts. The Wnt signaling antagonist, DKK2, is a direct downstream target of miR-483-3p. In the present study, miR-483-3p negatively regulated DKK2 protein levels. In human osteoblasts, DKK2 knockdown exerted an effect similar to that of miR-483-3p overexpression. DKK2 overexpression inhibited cell viability, DNA synthesis capacity and osteogenesis. DDK2 overexpression also decreased the Wnt1, $\beta$-catenin and cyclin D1 
A

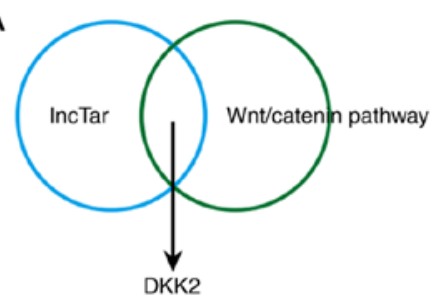

B

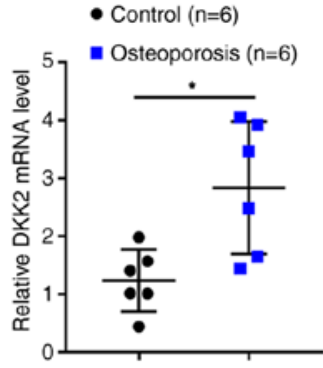

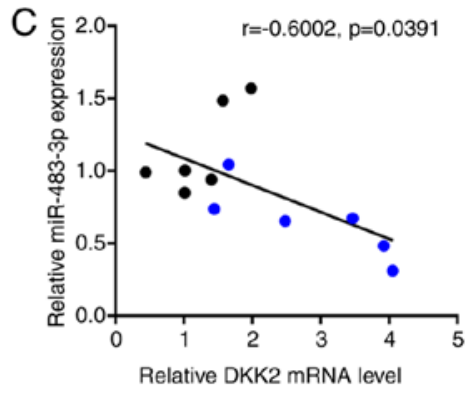

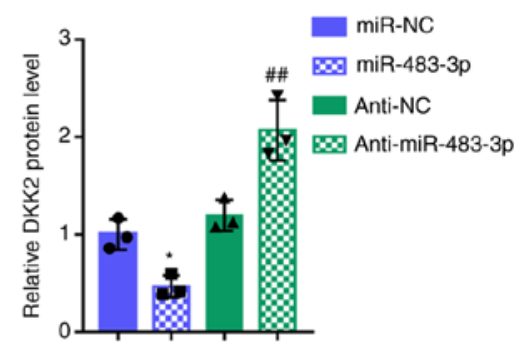

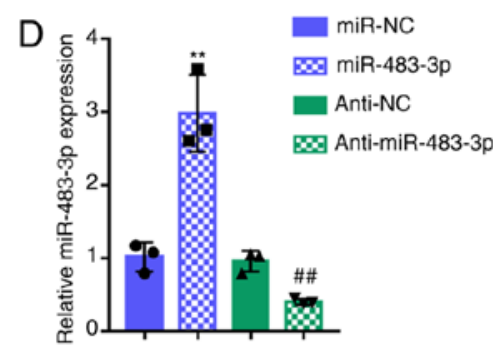
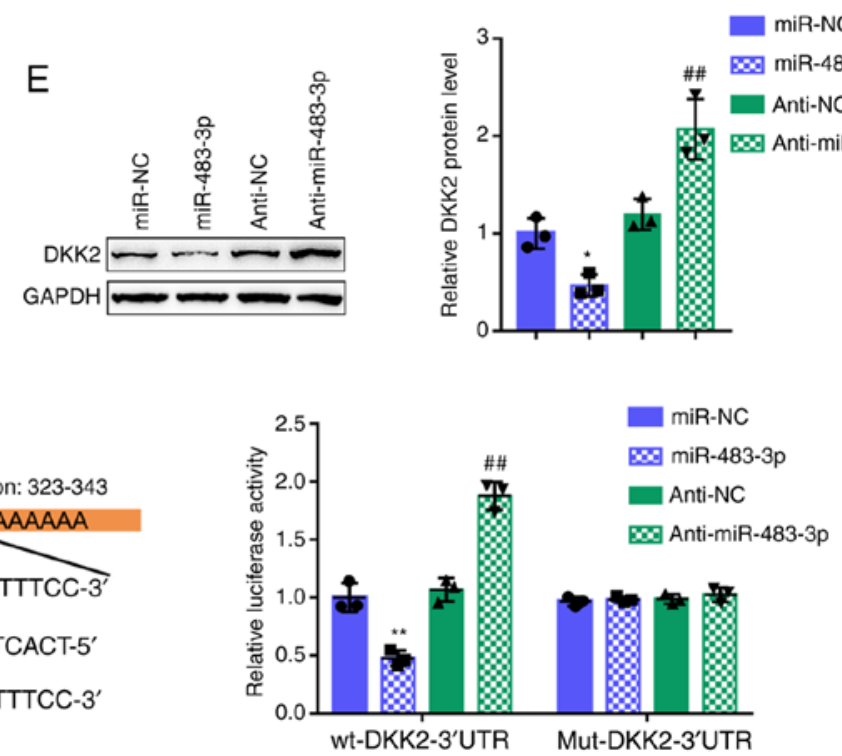

$\mathrm{F}$

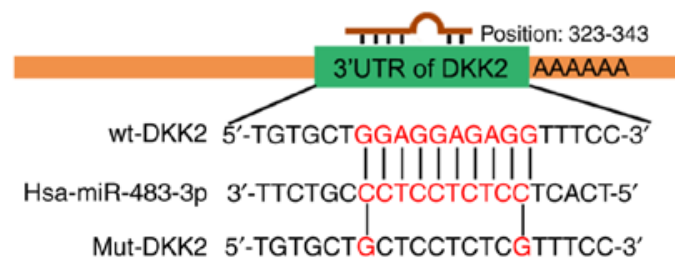

wt-DKK2-3'UTR Mut-DKK2-3'UTR

Figure 3. DKK2 is a direct downstream target of miR-483-3p. (A) The lncTar online tool was used to predict the downstream targets of miR-483-3p within Wnt/ $\beta$-catenin signaling-related genes, and DKK2 was predicted as a direct downstream target of miR-483-3p. (B) The expression of DKK2 in 6 normal bone tissues and 6 osteoporotic bone tissues was determined by RT-qPCR. (C) Correlation between miR-483-3p and DKK2 expression in tissue samples was analyzed by Pearson's correlation analysis. (D) miR-483-3p overexpression or inhibition was generated in hFOB1.19 cells by transfection with miR-483-3p or anti-miR-483-3p, as confirmed by RT-qPCR. (E) hFOB1.19 cells were transfected with miR-483-3p or anti-miR-483-3p and examined for DKK2 protein levels by western blot analysis. (F) Luciferase reporter assay was performed by constructing wild-type and mutant DKK2 3'-UTR reporter vectors as described in the 'Materials and methods'. These vectors were co-transfected into 293T cells with miR-483-3p or anti-miR-483-3p and the luciferase activity was determined. ${ }^{*} \mathrm{P}<0.05,{ }^{* *} \mathrm{P}<0.01$, compared to control or miR-NC group; ${ }^{\# \prime} \mathrm{P}<0.01$, compared to anti-NC group.

protein levels, as well as the RANKL/OPG ratio in human osteoblasts, and it promoted human osteoblast apoptosis. Thus, these finding demonstrate that DKK2 overexpression attenuates the functions of miR-483-3p overexpression, suggesting that miR-483-3p exerts its effects through DKK2 and the Wnt/ $\beta$-catenin pathway.

The role of miRNAs in osteogenesis has been widely investigated. Various miRNAs, such as miR-98, miR-335, miR-433 and miR-542, have been implicated in the promotion of osteogenesis. In particular, miR-98 binds to SOST mRNA. leading to osteoblast differentiation (24). miR-335 and miR-433 expression bind to Dkk1 mRNA in its 3 'UTR, thus promoting osteoblastogenesis $(25,26)$. During the process of osteoblast differentiation, miR-335 is highly expressed in pre-osteoblasts, thereby promoting the response of these cells to the Wnt pathway and enhancing osteogenesis (26). miR-542 promotes osteogenesis by targeting sFRP1 mRNA. In rats, the expression of miR-542, which prevents ovariectomy (OVX)-induced osteoporosis, is decreased in post-menopausal osteoporotic patients, suggesting that miR-542 contributes to the pathological processes of osteoporosis (27). Of note, it has been reported that miR-483-5p may target IGF2 to promote osteogenic differentiation, thus indicating its involvement in osteoporosis pathology (28). The present study demonstrated that miR-483-3p expression was inhibited in osteoporotic bone tissue samples, whereas it enhanced in osteogenically differentiated BSMCs, suggesting that miR-483-3p is involved in osteogenesis.

As was expected, miR-483-3p overexpression enhanced cell viability and DNA synthesis capacity, but suppressed cell apoptosis. Moreover, miR-483-3p overexpression increased the Ki-67 protein level, but decreased the cleaved-caspase-3/caspase-3 ratio. As is well known, Ki-67 is a nuclear protein associated with and necessary for cell proliferation. The inactivation of $\mathrm{Ki}-67$ results in the suppression of ribosomal RNA synthesis (29). Thus, Ki-67 is used as a proliferating cell marker (30). As regards caspase-3, it is the most important executioner caspase. Once the apoptotic signaling events, either extrinsic (death ligand) or intrinsic (mitochondrial), occur, caspase-3 is cleaved (31), which kills cells indiscriminately (32). Therefore, the upregulation of the cleaved-caspase-3/caspase-3 ratio is a reliable marker of apoptotic cells. In the present study, miR-483-3p overexpression 

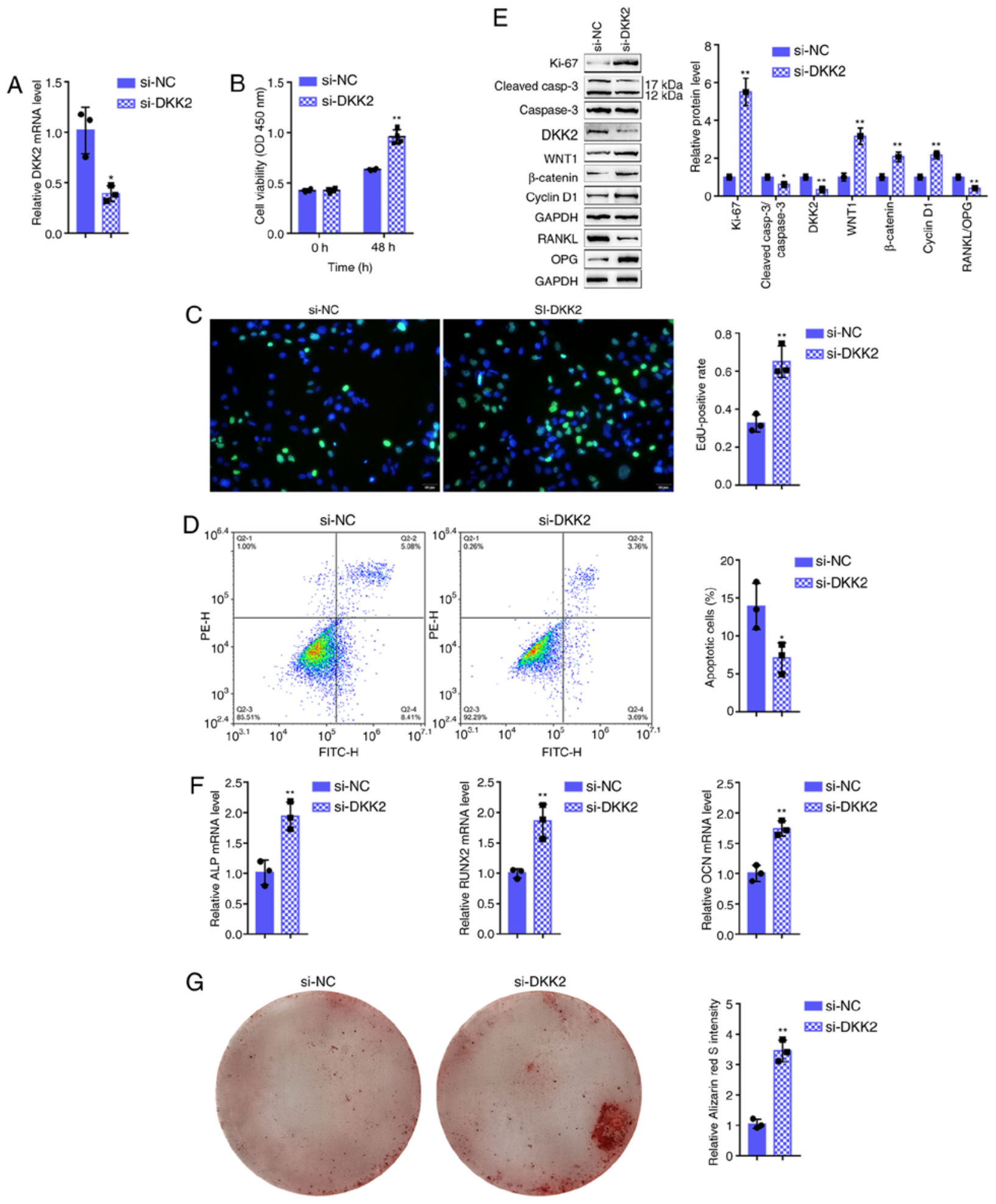

Figure 4. Effects of DKK2 on hFOB1.19 cell proliferation and osteogenic differentiation. (A) DKK2 knockdown was generated in hFOB1.19 cells by transfection with si-DKK2, as confirmed by RT-qPCR. hFOB1.19 cells were transfected with si-DKK2 and examined for (B) cell viability by CCK-8 assay; (C) DNA synthesis capacity by EdU assay, and (D) cell apoptosis by flow cytometric assay. (E) The protein levels of Ki-67, cleaved-caspase-3, caspase-3, WNT1, $\beta$-catenin, cyclin D1, RANKL and OPG were determined by western blot analysis, and (F) the mRNA expression of osteogenesis markers, including ALP, RUNX2, OCN and OPN, was determined by RT-qPCR. (G) The mineralization of hFOB1.19 cells was determined by Alizarin Red staining. "P<0.05, ${ }^{* *} \mathrm{P}<0.01$.

increased Ki-67 expression, whereas it decreased the cleaved-caspase-3/caspase-3 ratio, further indicating that miR-483-3p overexpression enhances the capacity of human osteoblasts to proliferate.

Human osteoblast proliferation, pre-osteoblast differentiation into mature osteoblasts and new bone matrix formation via mature osteoblasts are all critical steps of bone formation by osteoblasts. In the present study, miR-483-3p overexpression upregulated the mRNA expression of osteogenesis markers, including ALP, RUNX2 and OCN, and it increased the Wnt1, $\beta$-catenin and cyclin D1 protein levels. ALP is a typical protein marker of osteoblast-phenotype and osteogenic differentiation, 

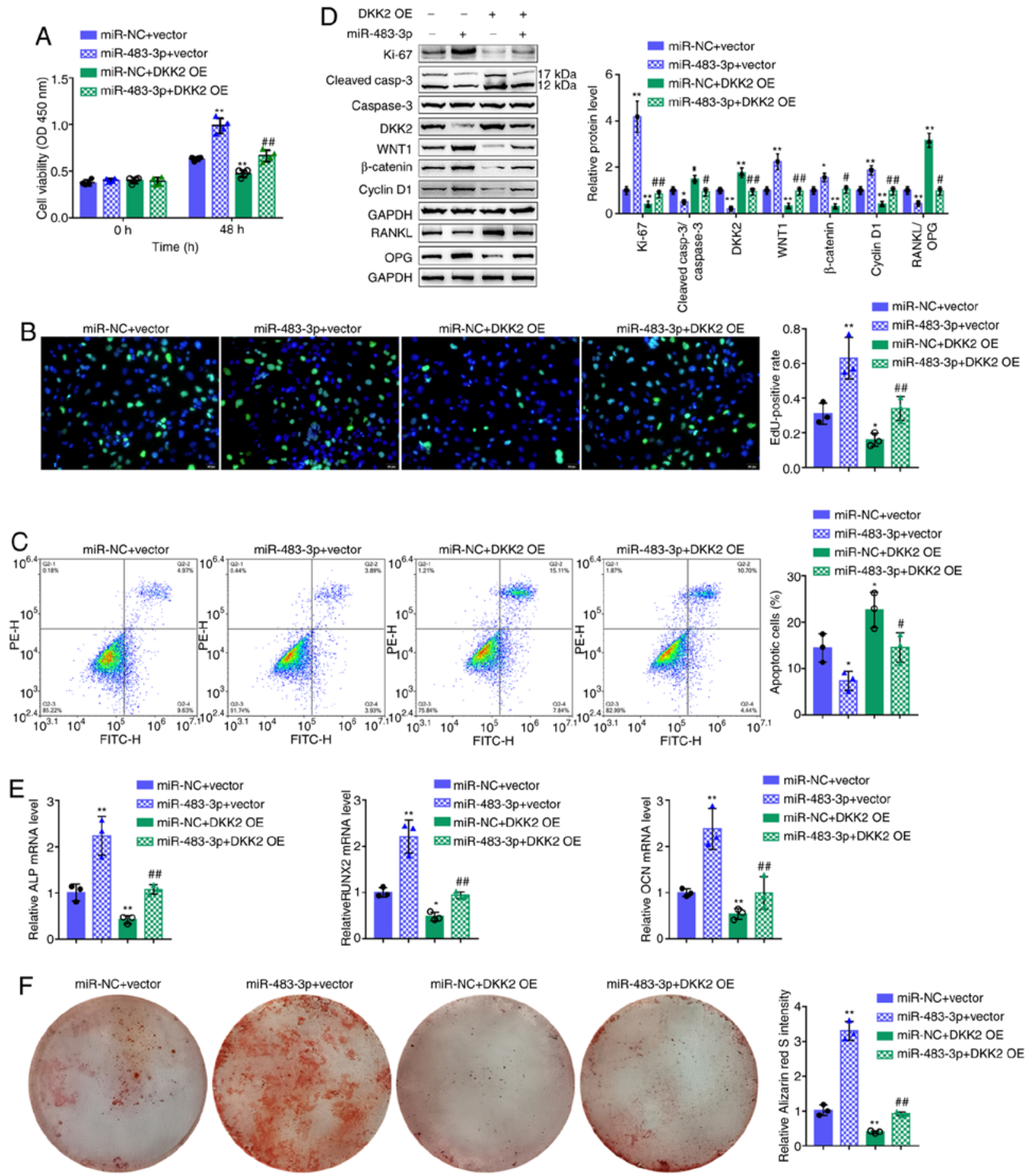

Figure 5. Dynamic effects of miR-483-3p and DKK2 on hFOB1.19 cell proliferation and osteogenic differentiation of hFOB1.19 cells co-transfected with miR-483-3p and DKK2 OE and examined for (A) cell viability by CCK-8 assay; (B) DNA synthesis capacity by EdU assay and (C) cell apoptosis by flow cytometry assay. (D) The protein levels of Ki-67, cleaved-caspase-3, caspase-3, DKK2, WNT1, $\beta$-catenin, cyclin D1, RANKL and OPG were detected by western blot analysis, and (E) the mRNA expression of osteogenesis markers, including ALP, RUNX2, OCN and OPN were determined by RT-qPCR. (F) The mineralization of hFOB1.19 cells was determined by Alizarin Red staining. ${ }^{*} \mathrm{P}<0.05,{ }^{* *} \mathrm{P}<0.01$, compared to the control group; ${ }^{\#} \mathrm{P}<0.05,{ }^{\# \#} \mathrm{P}<0.01$, compared to the miR-NC + DKK2 OE group.

and ALP activity is the most well-known biochemical marker of osteoblast activity. The osteoblast phenotype is characterized by two stages. In stage 1, ALP is detected during cell proliferation and matrix maturation $(5,33)$. In stage 2 , matrix mineralization occurs and late bone markers, such as OCN, are acquired. Multiple anabolic signaling pathways, such as Wnt and RUNX2 pathways, play positive roles in regulating bone formation (33). In addition, RANKL and OPG are two vital factors produced by osteoblasts which modulate the maturation and differentiation of osteoclasts, which regulate bone resorption (34). In the present study, as miR-483-3p overexpression increased the mRNA or protein expression of these key factors, it was inferred that the overexpression of miR-483-3p not only promotes osteoblast proliferation, but also significantly promotes the differentiation of pre-osteoblasts into mature osteoblasts and new bone matrix formation 
via mature osteoblasts, thereby enhancing bone formation by osteoblasts.

As regards the molecular mechanisms, the positive miR-483-3p regulation of Wnt $1, \beta$-catenin and cyclin D1 suggests that miR-483-3p may target Wnt signaling antagonist(s) to exert a promoting effect on Wnt signaling. The Wnt signaling pathway is regulated by two main classes of modulators, which are classified based on their crosstalk with Wnt signaling or its receptors (35). Secreted frizzled-related proteins (SFRPs) belong to the class that not only bind to Wnt protein, but also antagonize Wnt signaling (36). The other class consists of the DKK protein family, which binds to the Wnt receptor complex $(37,38)$. In the present study, the IncTar online tool was used to search these antagonists for the potential direct downstream targets of miR-483-3p and DKK2 was identified as a potential downstream target. The experimental results confirmed the putative miR-483-3p binding to and the negative regulation of DKK2. Through interacting with LRPs, DKKs suppress canonical Wnt signaling (39). The elevated expression of DKK2 has been reported to induce an abnormal phenotype of osteoarthritic osteoblasts and poor in vitro mineralization (40). In the present study, consistent with previous observations (34), the knockdown of DKK2 in human osteoblasts led to enhanced cell proliferation and enhanced osteogenesis, while the overexpression of DKK2 led to opposite results. More importantly, the overexpression of DKK2 significantly attenuated the promoting effects of miR-483-3p overexpression on osteoblast proliferation and osteogenesis, suggesting that miR-483-3p exerts its effects by targeting DKK2.

In conclusion, these findings demonstrate that the miR-483-3p/DKK2 axis modulates the osteoblast-mediated bone formation process by regulating osteoblast proliferation, pre-osteoblast differentiation into mature osteoblasts, and new bone matrix formation via mature osteoblasts. As regards the shortcoming of the present study, the function of the miR-483-3p/DKK2 axis warrants further in vivo and clinical investigations.

\section{Acknowledgements}

Not applicable.

\section{Funding}

The present study was supported by the Innovative Research and Development project of Hunan Development and Reform Commission (no. 2019412).

\section{Availability of data and materials}

All data generated or analyzed during this study are included in this published article or are available from the corresponding author on reasonable request.

\section{Authors' contributions}

$\mathrm{BZ}, \mathrm{KP}, \mathrm{GW}$ and $\mathrm{WC}$ were involved in the investigative aspects of the study. BZ and FC were involved in the writing of the manuscript. FC and YK were involved the design of the study.
PL and FC were involved in data collection and analysis. All authors have read and approved the final manuscript.

\section{Ethics approval and consent to participate}

Written informed consents were obtained from all subjects. All clinical sampling was performed with the approval of the Institutional Review Board and Ethics Committee of the Second Xiangya Hospital [approval no. 2019(202)].

\section{Patient consent for publication}

Not applicable.

\section{Competing interests}

The authors declare that they have no competing interests.

\section{References}

1. Armas LA and Recker RR: Pathophysiology of osteoporosis: New mechanistic insights. Endocrinol Metab Clin North Am 41: 475-486, 2012.

2. Bidwell JP, Alvarez MB, Hood M Jr and Childress P: Functional impairment of bone formation in the pathogenesis of osteoporosis: The bone marrow regenerative competence. Curr Osteoporos Rep 11: 117-125, 2013.

3. Khosla S, Melton LJ III and Riggs BL: The unitary model for estrogen deficiency and the pathogenesis of osteoporosis: Is a revision needed? J Bone Miner Res 26: 441-451, 2011.

4. Xie Z, Chen Y, Gurbuz S, Zhang B, Li Y, Bai F and Chen Y: Effects of teriparatide in Chinese and Caucasian women with osteoporosis: Bridging study on efficacy. Clin Interv Aging 14: 959-968, 2019.

5. An J, Yang H, Zhang Q, Liu C, Zhao J, Zhang L and Chen B: Natural products for treatment of osteoporosis: The effects and mechanisms on promoting osteoblast-mediated bone formation. Life Sci 147: 46-58, 2016.

6. Komori T: Signaling networks in RUNX2-dependent bone development. J Cell Biochem 112: 750-755, 2011.

7. Guntur AR and Rosen CJ: IGF-1 regulation of key signaling pathways in bone. Bonekey Rep 2: 437, 2013.

8. Kobayashi Y, Uehara S, Udagawa N and Takahashi N: Regulation of bone metabolism by Wnt signals. J Biochem 159: 387-392, 2016.

9. Takahashi N, Maeda K, Ishihara A, Uehara S and Kobayashi Y: Regulatory mechanism of osteoclastogenesis by RANKL and Wnt signals. Front Biosci (Landmark Ed) 16: 21-30, 2011.

10. Landgraf P, Rusu M, Sheridan R, Sewer A, Iovino N, Aravin A, Pfeffer S, Rice A, Kamphorst AO, Landthaler M, et al: A mammalian microRNA expression atlas based on small RNA library sequencing. Cell 129: 1401-1414, 2007.

11. Sun X, Guo Q, Wei W, Robertson S, Yuan Y and Luo X: Current progress on MicroRNA-based gene delivery in the treatment of osteoporosis and osteoporotic fracture. Int J Endocrinol 2019: 6782653, 2019.

12. Liu H, Liu Q, Wu XP, He HB and Fu L: MiR-96 regulates bone metabolism by targeting osterix. Clin Exp Pharmacol Physiol 45: 602-613, 2018

13. Xia Z, Chen C, Chen P, Xie H and Luo X: MicroRNAs and their roles in osteoclast differentiation. Front Med 5: 414-419, 2011.

14. Kapinas K, Kessler C, Ricks T, Gronowicz G and Delany AM: MiR-29 modulates Wnt signaling in human osteoblasts through a positive feedback loop. J Biol Chem 285: 25221-25231, 2010.

15. Hassan MQ, Maeda Y, Taipaleenmaki H, Zhang W, Jafferji M, Gordon JA, Li Z, Croce CM, van Wijnen AJ, Stein JL, et al: MiR-218 directs a Wnt signaling circuit to promote differentiation of osteoblasts and osteomimicry of metastatic cancer cells. J Biol Chem 287: 42084-42092, 2012.

16. Zhang WB, Zhong WJ and Wang L: A signal-amplification circuit between miR-218 and Wnt//-catenin signal promotes human adipose tissue-derived stem cells osteogenic differentiation. Bone 58: 59-66, 2014. 
17. Xu X, Jiang H, Li X, Wu P, Liu J, Wang T, Zhou X, Xiong J and $\mathrm{Li} \mathrm{W}$ : Bioinformatics analysis on the differentiation of bone mesenchymal stem cells into osteoblasts and adipocytes. Mol Med Rep 15: 1571-1576, 2017.

18. Martin EC, Qureshi AT, Dasa V, Freitas MA, Gimble JM and Davis TA: MicroRNA regulation of stem cell differentiation and diseases of the bone and adipose tissue: Perspectives on miRNA biogenesis and cellular transcriptome. Biochimie 124: 98-111,2016.

19. Tornero-Esteban P, Rodriguez-Rodriguez L, Abasolo L, Tomé M, López-Romero P, Herranz E, González MA, Marco F, Moro E, Fernández-Gutiérrez B and Lamas JR: Signature of microRNA expression during osteogenic differentiation of bone marrow MSCs reveals a putative role of miR-335-5p in osteoarthritis BMC Musculoskelet Disord 16: 182, 2015.

20. Xie F, Zhou B, Wang J, Liu T, Wu X, Fang R, Kang Y and Dai R: Microstructural properties of trabecular bone autografts: Comparison of men and women with and without osteoporosis Arch Osteoporos 13: 18, 2018.

21. Lu J, Yang J, Zheng Y, Chen X and Fang S: Extracellular vesicles from endothelial progenitor cells prevent steroid-induced osteoporosis by suppressing the ferroptotic pathway in mouse osteoblasts based on bioinformatics evidence. Sci Rep 9: 16130, 2019.

22. Livak KJ and Schmittgen TD: Analysis of relative gene expression data using real-time quantitative PCR and the 2(-Delta Delta C(T)) method. Methods 25: 402-408, 2001.

23. Zhang F, Cao K, Du G, Zhang Q and Yin Z: MiR-29a promotes osteoblast proliferation by downregulating DKK-1 expression and activating Wnt $/ \beta$-catenin signaling pathway. Adv Clin Exp Med 28: 1293-1300, 2019.

24. Ye W, Wang Y, Mei B, Hou S, Liu X, Wu G, Qin L, Zhao K and Huang Q: Computational and functional characterization of four SNPs in the SOST locus associated with osteoporosis. Bone 108: 132-144, 2018

25. Tang X, Lin J, Wang G and Lu J: MicroRNA-433-3p promotes osteoblast differentiation through targeting DKK1 expression. PLoS One 12: e0179860, 2017.

26. Zhang J, Tu Q, Bonewald LF, He X, Stein G, Lian J and Chen J: Effects of miR-335-5p in modulating osteogenic differentiation by specifically downregulating Wnt antagonist DKK1. J Bone Miner Res 26: 1953-1963, 2011.

27. Zhang X, Zhu Y, Zhang C, Liu J, Sun T, Li D, Na Q, Xian CJ, Wang L and Teng Z: MiR-542-3p prevents ovariectomy-induced osteoporosis in rats via targeting SFRP1. J Cell Physiol 233: 6798-6806, 2018.

28. Li K, Chen S, Cai P, Chen K, Li L, Yang X, Yi J, Luo X, Du Y and Zheng H: MiRNA-483-5p is involved in the pathogenesis of osteoporosis by promoting osteoclast differentiation. Mol Cell Probes 49: 101479, 2020
29. Rahmanzadeh R, Huttmann G, Gerdes J and Scholzen T: Chromophore-assisted light inactivation of pKi-67 leads to inhibition of ribosomal RNA synthesis. Cell Prolif 40: 422-430, 2007.

30. Scholzen T and Gerdes J: The Ki-67 protein: From the known and the unknown. J Cell Physiol 182: 311-322, 2000.

31. Boatright KM and Salvesen GS: Mechanisms of caspase-activation. Curr Opin Cell Biol 15: 725-731, 2003.

32. Ghavami S, Hashemi M, Ande SR, Yeganeh B, Xiao W, Eshraghi M, Bus CJ, Kadkhoda K, Wiechec E, Halayko AJ and Los M: Apoptosis and cancer: Mutations within caspase-genes. J Med Genet 46: 497-510, 2009.

33. Franceschi RT, Ge C,Xiao G, Roca H and Jiang D: Transcriptional regulation of osteoblasts. Ann NY Acad Sci 1116: 196-207, 2007.

34. Ren H, Ren H, Li X, Yu D, Mu S, Chen Z and Fu Q: Effects of intermedin on proliferation, apoptosis and the expression of OPG/RANKL/M-CSF in the MC3T3-E1 osteoblast cell line. Mol Med Rep 12: 6711-6717, 2015.

35. Sharma G, Sharma AR, Seo EM and Nam JS: Genetic polymorphism in extracellular regulators of Wnt signaling pathway. Biomed Res Int 2015: 847529, 2015.

36. Kawano Y and Kypta R: Secreted antagonists of the Wnt signalling pathway. J Cell Sci 116: 2627-2634, 2003.

37. Caraci F, Busceti C, Biagioni F, Aronica E, Mastroiacovo F, Cappuccio I, Battaglia G, Bruno V, Caricasole A, Copani A and Nicoletti F: The Wnt antagonist, Dickkopf-1, as a target for the treatment of neurodegenerative disorders. Neurochem Res 33 2401-2406, 2008.

38. Veeck $\mathbf{J}$ and Dahl E: Targeting the Wnt pathway in cancer: The emerging role of Dickkopf-3. Biochim Biophys Acta 1825: 18-28, 2012.

39. Zorn AM: Wnt signalling: Antagonistic dickkopfs. Curr Biol 11: R592-R595, 2001.

40. Chan TF, Couchourel D, Abed E, Delalandre A, Duval N and Lajeunesse D: Elevated Dickkopf-2 levels contribute to the abnormal phenotype of human osteoarthritic osteoblasts. J Bone Miner Res 26: 1399-1410, 2011.

This work is licensed under a Creative Commons Attribution-NonCommercial-NoDerivatives 4.0 International (CC BY-NC-ND 4.0) License. 